ARTICLE

\title{
Serial protein crystallography in an electron microscope
}

Robert Bücker (10 1, Pascal Hogan-Lamarre ${ }^{1,2}$, Pedram Mehrabi (iD) 1, Eike C. Schulz (10 1, Lindsey A. Bultema1, Yaroslav Gevorkov (10 ${ }^{3,4}$, Wolfgang Brehm³ , Oleksandr Yefanov ${ }^{3}$, Dominik Oberthür ${ }^{3}$, Günther H. Kassier ${ }^{1} \&$ R.J. Dwayne Miller (i) $1,2 \bowtie$

Serial X-ray crystallography at free-electron lasers allows to solve biomolecular structures from sub-micron-sized crystals. However, beam time at these facilities is scarce, and involved sample delivery techniques are required. On the other hand, rotation electron diffraction (MicroED) has shown great potential as an alternative means for protein nanocrystallography. Here, we present a method for serial electron diffraction of protein nanocrystals combining the benefits of both approaches. In a scanning transmission electron microscope, crystals randomly dispersed on a sample grid are automatically mapped, and a diffraction pattern at fixed orientation is recorded from each at a high acquisition rate. Dose fractionation ensures minimal radiation damage effects. We demonstrate the method by solving the structure of granulovirus occlusion bodies and lysozyme to resolutions of $1.55 \AA$ and $1.80 \AA$, respectively. Our method promises to provide rapid structure determination for many classes of materials with minimal sample consumption, using readily available instrumentation.

\footnotetext{
${ }^{1}$ Max Planck Institute for the Structure and Dynamics of Matter, CFEL, Luruper Chaussee 149, 22761 Hamburg, Germany. ${ }^{2}$ Departments of Chemistry and Physics, University of Toronto, 80 St. George Street, Toronto, ON M5S 3H6, Canada. ${ }^{3}$ Center for Free-Electron Laser Science, DESY, Notkestrasse 85, 22607 Hamburg, Germany. ${ }^{4}$ Institute of Vision Systems, Hamburg University of Technology, Harburger Schlossstrasse 20, 21079 Hamburg, Germany.

$凶_{\text {email: dwayne.miller@mpsd.mpg.de }}$
} 
$\mathrm{n}$ understanding of macromolecular structure is crucial for insight into the function of complex biological systems. Despite recent advances in single-particle cryoelectron microscopy (cryo-EM), the vast majority of highresolution structures are determined by crystallographic methods (http://www.rcsb.org/stats/summary). This includes the majority of membrane proteins, which are often too small for computational alignment as required by single-particle analysis $^{1,2}$. An important limitation of biomolecular crystallography lies in the difficulty to obtain large, well-ordered crystals, which is particularly prevalent for membrane proteins and macromolecular complexes. Sub-micron crystals can be obtained more readily and are a common natural phenomenon, but often escape structure determination as the small diffracting volume and low tolerated dose of typically tens of $\mathrm{MGy}^{3,4}$ prohibit the measurement of sufficient signal. However, during the past few years crystallographic techniques have emerged that are able to exploit nanocrystals for diffraction experiments. Notably, X-ray free-electron lasers (XFELs) have driven the development of serial crystallography ${ }^{5-10}$, a technique that is also increasingly applied at synchrotron sources ${ }^{11-17}$. Here, acquiring snapshots in a single orientation from each crystal instead of a rotation series avoids dose accumulation, permitting higher fluences, which concomitantly decreases the required diffracting volume. Sufficient signal-to-noise ratio and completeness is achieved through merging of many thousands of such snapshots. Ideally, radiationdamage effects are entirely evaded either by a "diffract-beforedestroy" mode using femtosecond XFEL pulses ${ }^{5}$ or by imposing doses too low to cause significant structural damage of each crystal, which has also been implemented at synchrotron microfocus beam lines ${ }^{11,12,18}$. However, the scarcity and costliness of XFEL beamtime limits the use of protein nanocrystals for routine structure determination. The development of serial crystallography using smaller scale, ideally laboratory-based instrumentation is therefore highly desirable.

Electron microscopes are a comparatively ubiquitous and costeffective alternative for measuring diffraction from nanocrystals. While the low penetration depth of electrons renders them unsuitable for large three-dimensional crystals, their physical scattering properties are specifically advantageous for sub-micron crystals of radiation-sensitive materials. Compared to X-rays, the obtainable diffraction signal for a given crystal volume and tolerable radiation dose is up to three orders of magnitude larger due to the higher ratio of elastic to inelastic electron scattering events and a much smaller energy deposition per inelastic event ${ }^{1,19}$. While seminal experiments on 2D crystals ${ }^{20}$ were restricted to a small class of suitable samples, various successful implementations of 3D rotation electron diffraction (3D ED) solving structures of beam-sensitive small molecules ${ }^{21-23}$ sparked interest in applying 3D crystallography also to biomolecules, a technique also referred to as MicroED ${ }^{24-26}$. Several research groups have now succeeded in solving protein structures by merging ED data from as little as one up to a few sub-micron-sized vitrified protein crystals using rotation diffraction ${ }^{27-30}$, and very recently the first unknown protein structure could be determined ${ }^{31}$. Automated procedures are becoming increasingly available to reduce the manual effort of identifying suitable crystals, acquiring rotation series while keeping the crystal under the beam, and sequentially addressing many crystals to be merged ${ }^{32-36}$. However, despite the high dose efficiency of electrons, damage accumulation throughout the rotation series remains a limiting factor, and acquisition as well as sample screening require careful operation at extremely low dose rates ${ }^{37}$. Recently, a serial ED (SerialED) scheme has been introduced for small-molecule crystals, where, similar to the aforementioned Xray experiments, still-diffraction snapshots were obtained and used for structure determination ${ }^{38}$.
Here we apply SerialED to protein nanocrystals, using a doseefficient automated data collection scheme that enabled us to solve the highest-resolution protein structure by ED to date. This method provides a viable alternative to serial femtosecond crystallography for the determination of high-resolution protein structures from sub-micron-sized crystals using laboratory-based instrumentation.

\section{Results}

STEM-based SerialED. We perform protein crystallography by SerialED using a parallel nanobeam in a scanning transmission electron microscope (S/TEM). Analogous to the approach of serial X-ray crystallography, we mitigate the problem of damage accumulation by exposing each crystal only once with a high degree of automation and ease of use. A recently developed indexing algorithm ${ }^{39}$ allows crystal orientation to be determined followed by merging into a full crystallographic data set. Our SerialED approach operates on crystals randomly dispersed on a TEM grid and consists of two automated steps. First, the sample is moved to a previously unexposed grid region and an arbitrary, fixed goniometer tilt angle is chosen. An overview image of a TEM grid region is recorded in scanning (STEM) mode at a negligible radiation dose $(\approx 5 \%$ of that later used for diffraction acquisition), and the positions of the crystals are automatically mapped $^{32,40}$ (Fig. 1a). Second, still ED patterns are recorded from each (randomly oriented) crystal, synchronizing the microscope's beam deflectors with a high frame rate camera (Fig. 1b). No sample rotation is performed. Thereby, a hit fraction approaching $100 \%$ with a peak data collection rate of up to thousands of diffraction patterns per second can be achieved. While the former is defined by the accuracy of the mapping algorithm used to identify crystals in the STEM overview image, the latter is limited only by source brightness and camera frame rate. After completion of the diffraction acquisition, the sample is moved to a fresh region, and the sequence is repeated until sufficiently many diffraction patterns have been collected. Importantly, no special sample delivery devices are required, since the full workflow is conducted in a conventional S/TEM or dedicated STEM instrument. The nanobeam diameter can be matched to the typical crystal size of the sample under study by choosing an appropriate condenser aperture and microscope probe mode, minimizing background scattering and diffraction from multiple lattices.

Granulovirus occlusion bodies. As a test system, we have chosen natively grown, vitrified granulovirus particles with crystalline occlusion bodies (OBs) (granulin). The particle size of $\sim 100 \times$ $100 \times 300 \mathrm{~nm}^{3}$ and morphological homogeneity makes this system an ideal target for serial nanocrystallography. Furthermore, granulovirus has previously been studied at LCLS ${ }^{7}$, and is therefore well suited for purposes of comparing the SerialED approach to XFEL data. We acquired $\sim 32,000$ diffraction patterns from a total sample area of $0.036 \mathrm{~mm}^{2}$ on a vitrified TEM grid within a $4 \mathrm{~h}$ net measurement duration, that is, including auxiliary steps such as manual search for suitable grid regions containing a large number of viruses embedded in a sufficiently thin ice layer, acquisition of mapping images, and automatic crystal identification. Within each grid region, we achieved an average hit fraction of $69 \%$ at an acquisition rate of $\approx 50 \mathrm{~Hz}$ (see section on dose fractionation below and Supplementary Fig. 2). Each crystal was measured in a single orientation, with the goniometer tilt occasionally changed between acquisition runs of different regions (up to $40^{\circ}$ ) to mitigate effects of preferred sample orientation. Of these hits, $81 \%$ could subsequently be indexed and used for merging into a full data set (Supplementary Fig. 4). We obtained a $100 \%$ complete data set and Coulomb potential maps 
a

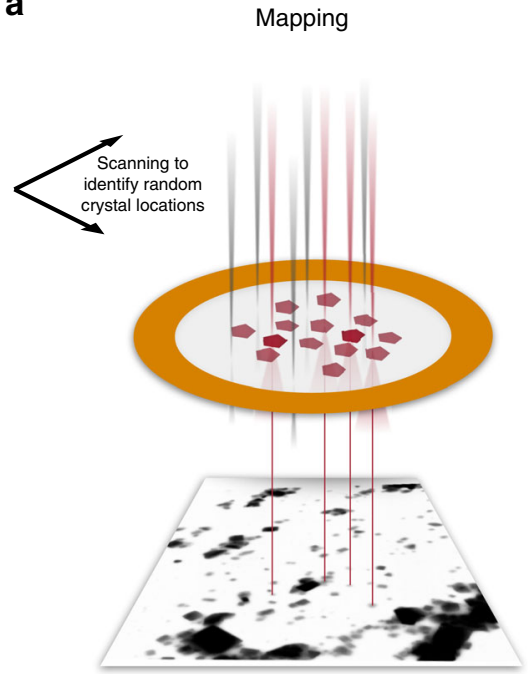

Map of crystal positions b

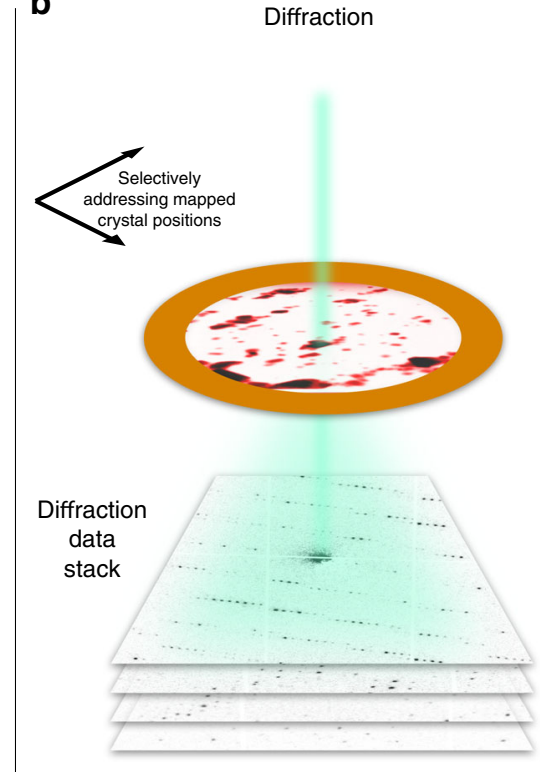

Fig. 1 Serial nanobeam electron diffraction scheme. a The sample is first mapped in low-dose STEM mode over a large region (typically $\approx 20 \mu \mathrm{m}$ edge length), yielding a real-space image. Crystals show up as clear features and can be identified automatically. $\mathbf{b}$ The beam of $\approx 100 \mathrm{~nm}$ diameter is sequentially steered to each found crystal position, and diffraction patterns are acquired at a rate of up to $1 \mathrm{kHz}$.

of excellent quality at $1.55 \AA$ resolution $\left(R_{\text {free }} / R_{\text {work }}=0.19 / 0.17\right)$, according to the $\mathrm{CC}^{\star}>0.5$ cut-off criterion ${ }^{41}$ (Figs. 2 and 3 and Table 1), improving on published XFEL $\operatorname{data}^{7}$ at $2.00 \AA$ resolution.

Lysozyme. Furthermore, we applied the SerialED method to the common test sample hen egg-white lysozyme (HEWL). HEWL crystals of typically $100-500 \mathrm{~nm}$ diameter were deposited on a standard TEM grid and vitrified (see Methods). Two independently prepared samples were measured in separate acquisition runs over a total measurement duration of $3 \mathrm{~h}$ and a sample area of $0.010 \mathrm{~mm}^{2}$. Diffraction patterns from 1325 nanocrystals were collected, achieving a hit fraction of $62 \%$ at an acquisition rate of $50 \mathrm{~Hz} .83 \%$ of the obtained patterns could be successfully indexed and used for merging (78\% completeness, see Supplementary Fig. 4), resulting in a Coulomb potential map of high quality to $1.8 \AA$ resolution (Fig. 2 and Table 1 ). The determined HEWL structure compares well to previously determined structures by $\mathrm{X}$-ray and microED techniques (see Methods).

Radiation damage and dose fractionation. The high frame rate and zero background of the detector applied in our experiment allows recording a burst series comprising several frames instead of a single snapshot for each crystal, yielding a dose-fractionated diffraction-during-destruction movie data stack. Both data sets shown were acquired with the camera running continuously at 500 frames/s; each crystal was exposed for 10 movie frames with the beam resting at the crystal position as determined in the mapping step, resulting in a net acquisition rate of $\approx 50 \mathrm{~Hz}$ (Supplementary Fig. 2). Therefore, the per-crystal exposure time of $20 \mathrm{~ms}$ was fractionated into a stack of diffraction patterns of $2 \mathrm{~ms}$ exposure time each, which exhibit a pronounced fading of high-resolution peaks (Fig. 3). A final set of diffraction images was generated by cumulatively summing movie frames in the acquired data. Thus, the effective integration time and dose per crystal can be chosen after data acquisition has concluded, trading off between low radiation-damage (short integration) and high signal-to-noise ratio (long integration). Hence, a priori knowledge of the sample's radiation sensitivity (critical dose) is not required, and data can be obtained before the onset of observable radiation damage. For our data sets, we find an instantly detectable loss of high-resolution Bragg peaks, in accordance with previous studies $^{37}$ (Fig. 3c). In Fig. 4c, mean reflection intensities from the granulin data set are shown for several resolution shells. Exponential fits to the data show a fair agreement and lead to estimated 1/e decay times of $14.9(4) \mathrm{ms}$ at $5.00 \AA, 6.8(3) \mathrm{ms}$ at $2.33 \AA$, 5.9 (3) $\mathrm{ms}$ at $1.96 \AA, 5.2(2) \mathrm{ms}$ at $1.75 \AA$, and 5.0(3) $\mathrm{ms}$ at $1.61 \AA$, the latter corresponding to an approximate dose of $\approx 2.6 \mathrm{e}^{-} / \AA^{2}$. The optimal integrated dose was found by observing the half-set correlation coefficient $\mathrm{CC}_{1 / 2} 41$ calculated for merged data sets that were derived from diffraction patterns summed over different numbers of movie frames (Fig. 3d). For granulin, optimal data quality was reached for summation of the first five movie frames, corresponding to an exposure time of $10 \mathrm{~ms}$, and an integrated dose of $\approx 4.7 \mathrm{e}^{-} / \AA^{2}$; for lysozyme, we found an optimal dose of $\approx 2.6 \mathrm{e}^{-} / \AA^{2}$. More detailed measurements of site-specific and global radiation-damage effects, as well as optimization of data acquisition and analysis strategies to further improve dose efficiency and resolution, will be the subject of future work.

\section{Discussion}

Our results show that SerialED allows the determination of protein structures at high resolution from extremely small protein crystals in a rapid, efficient, and automated manner. No sample rotation during measurement of each crystal is required, simplifying the measurement and allowing the use of higher doses for each diffraction pattern. Also, no manual screening and selection of individual suitable crystals under low-dose conditions are necessary. In contrast to wide-field TEM-based crystal mapping 36,38 , our STEM-based scheme neither requires frequent mode switching of the microscope (which always remains in diffraction mode) nor accurate beam-position calibrations, as crystal mapping and nanobeam positioning are achieved with the same set of deflectors. Furthermore, the acquisition speed is not limited by relatively slow softwarebased scripting of the microscope, which is entirely bypassed (see Methods), and a small condenser aperture can be used at all times, achieving a fully parallel (Köhler) nanobeam 
a

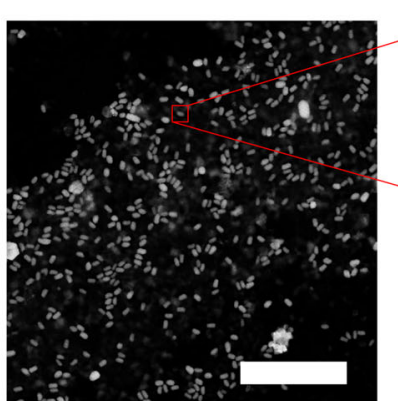

c
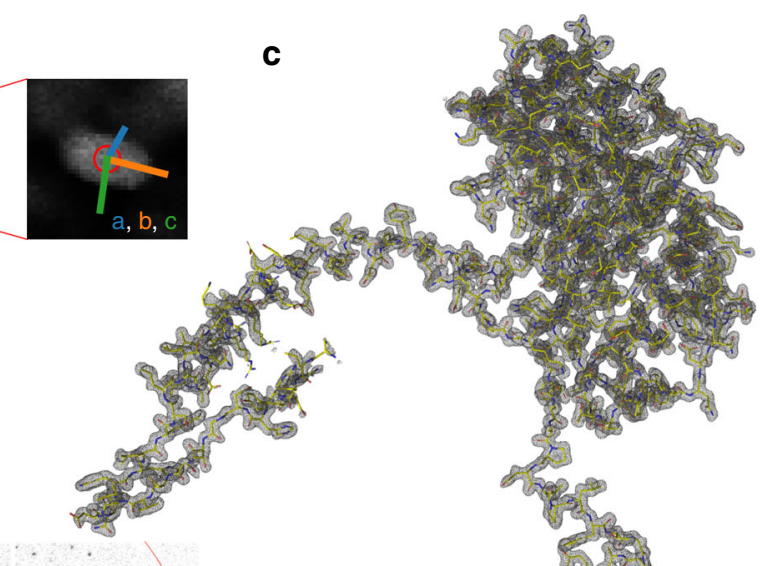

b

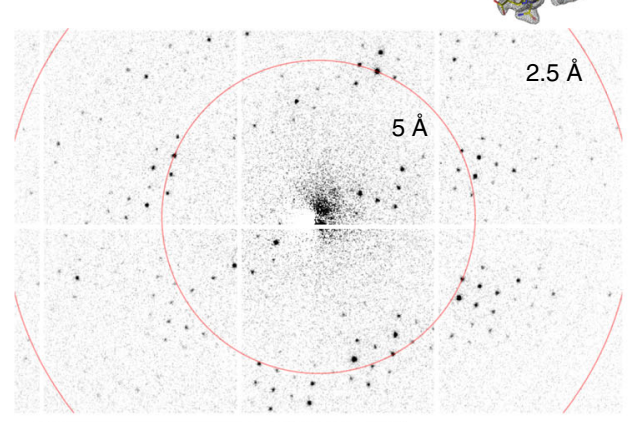

d

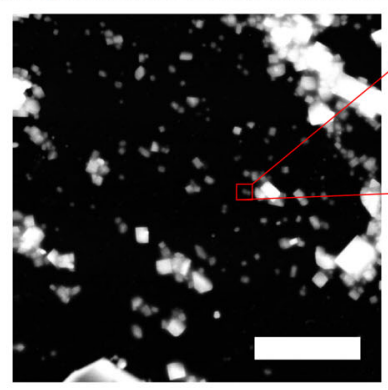

e
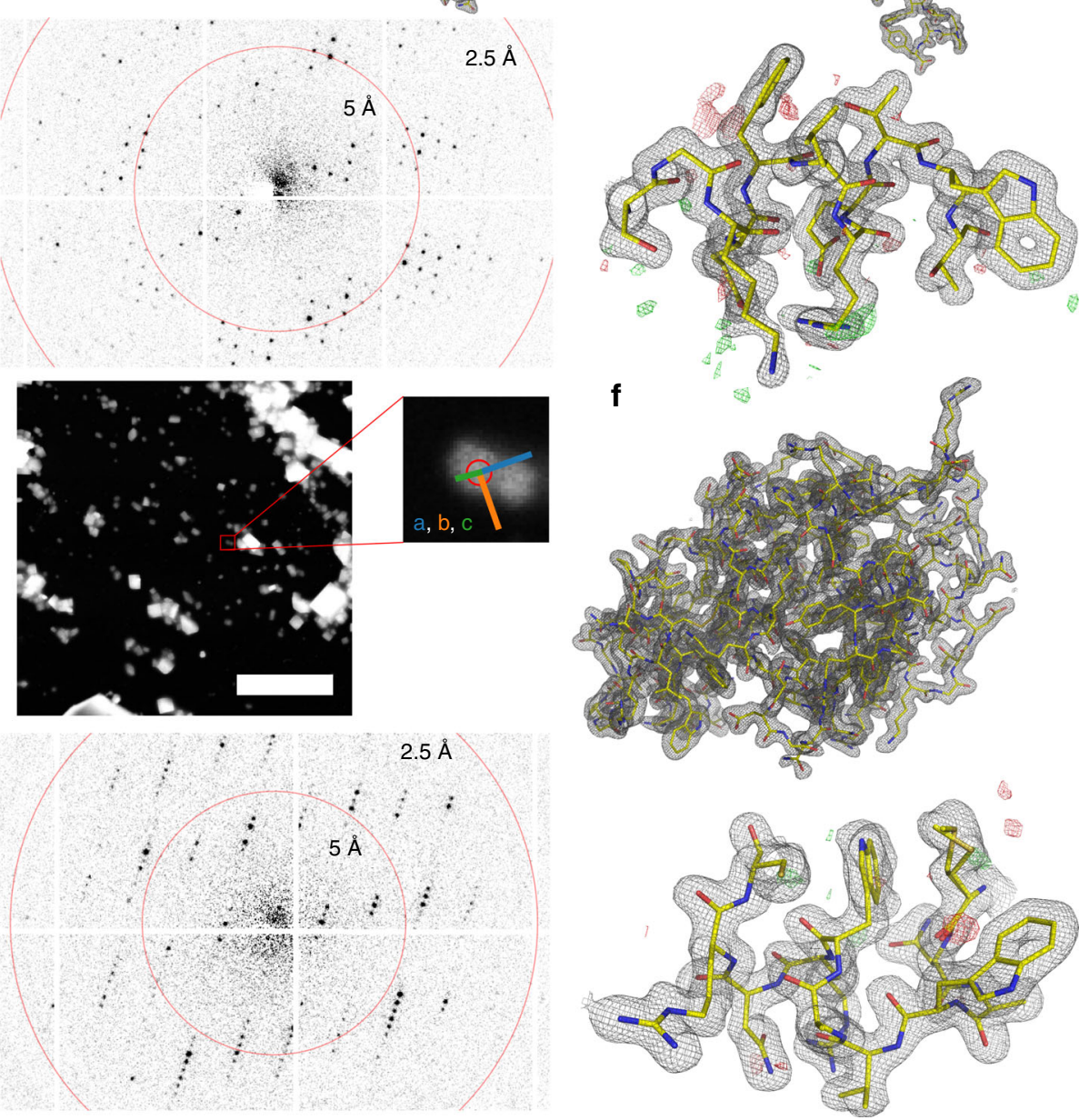

f

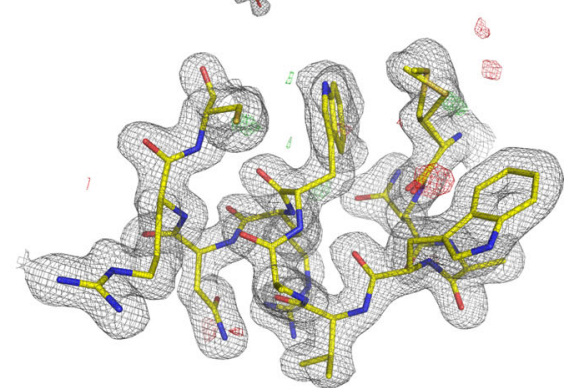

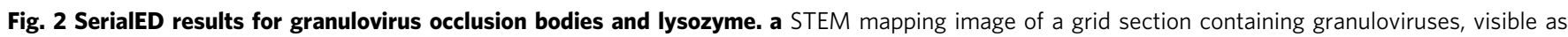
bright features (scale bar is $5 \mu \mathrm{m}$ ). A zoomed view of a representative virus is shown, where the red circle corresponds to the diffraction nanobeam diameter of $\approx 110 \mathrm{~nm}$. Colored lines indicate the lattice vectors found after indexing of the diffraction pattern. $\mathbf{b}$ Diffraction pattern acquired from the features shown in a. c Obtained structures of granulin; $2 F_{o}-F_{c}$ map of the entire structure, and zoom into a randomly chosen region, with $F_{o}-F_{c}$ map overlaid. The maps are at $1.55 \AA$ resolution and contoured at $\pm 1 \sigma$ and $\pm 3 \sigma$, respectively. $\mathbf{d}$-f Analogous for lysozyme nanocrystals; maps are at $1.8 \AA$ resolution.

illumination (Fig. 4), thus enabling distortion-free diffraction. Minimal-damage acquisition is ensured using a dosefractionated diffraction-during-destruction scheme and a posteriori critical dose determination. We have demonstrated a net acquisition rate of $35 \mathrm{~Hz}$ when factoring in the hit fraction (Supplementary Fig. 2), which is comparable to current liquidjet XFEL ${ }^{8,9}$ and synchrotron fixed-target ${ }^{11,12,18}$ experiments. Note that a further increase of more than an order of magnitude can be achieved if dose fractionation is omitted and acquisition speed becomes detector frame rate limited. A complication of
SerialED data analysis is the difficulty of determining space group and lattice parameters from single high-energy ED patterns due to the flatness of the Ewald sphere $(\lambda=0.025 \AA)$; successful indexing as demonstrated here requires prior knowledge of the crystal parameters. However, those can for instance be determined from an auxiliary low-resolution rotation diffraction data set obtained from few crystals on the same sample. Alternatively, an approach of clustering spot-distance data from all acquired patterns and deriving lattice parameters from comparison to forward modeling has yielded promising 
a

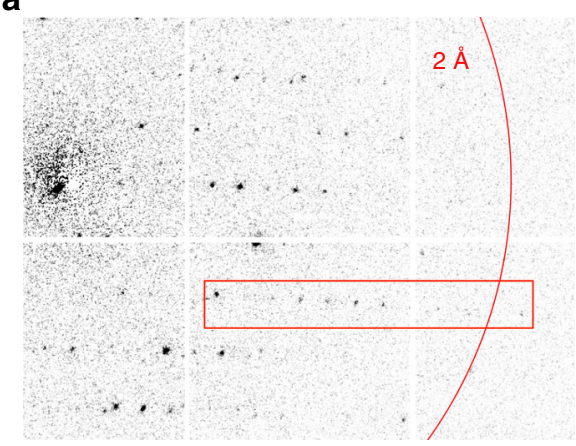

C

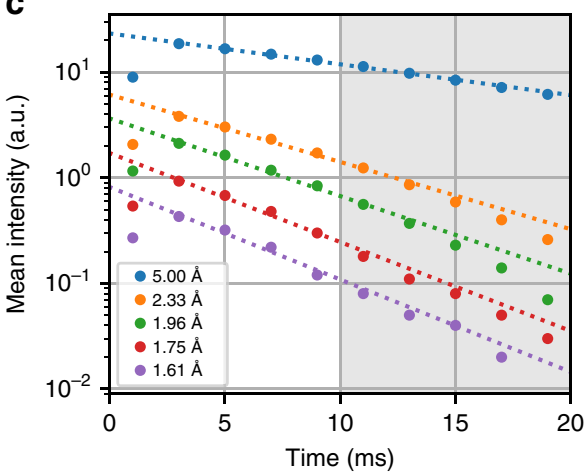

b

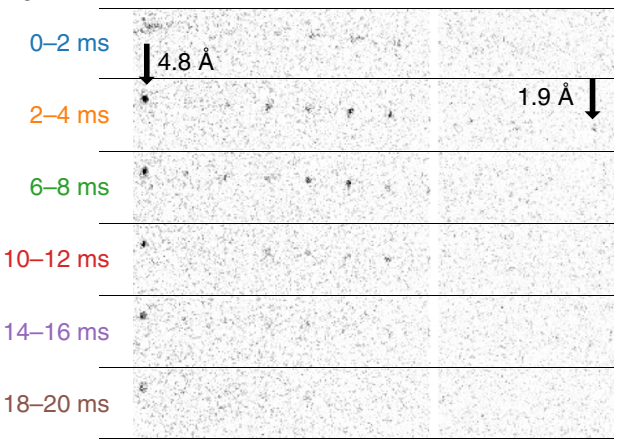

d

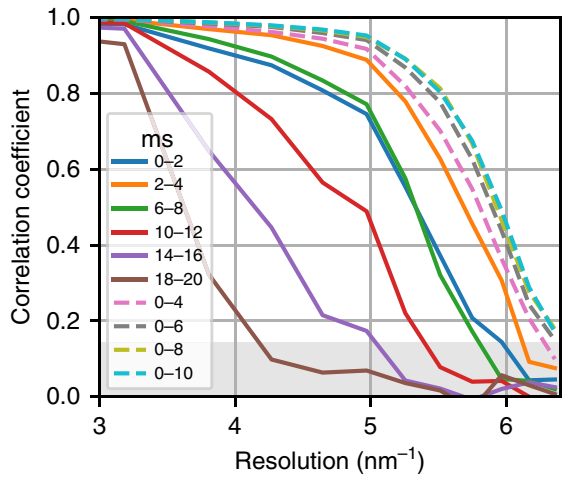

Fig. 3 Radiation damage during dose-fractionated acquisition. a Typical diffraction pattern from a granulovirus occlusion body. The red box indicates the enlarged region in $\mathbf{b}$. $\mathbf{b}$ Enlarged diffraction pattern section for several single frames from the dose-fractionated movie stack, each of 2 ms duration. The integration time of each frame relative to the beam first hitting the crystal is specified. Note the fading of the diffraction spots, especially at high resolutions. The first shot is affected by residual beam motion and hence has a shorter effective integration time and shows blurring artefacts. c Mean intensity of Bragg reflections for different resolution shells as a function of delay time, and exponential fit lines, where the first time point has been excluded from the fit. The shaded area corresponds to delay times beyond $10 \mathrm{~ms}$, which have been excluded from our data analysis. $\mathbf{d}$ Resolution-dependent correlation coefficients $C C_{1 / 2}$ shown from 3.33 to $1.55 \AA$ resolution. Solid lines correspond to single movie frames as in $\mathbf{b}$. Dashed lines correspond to images that were cumulatively summed over several frames. The shaded area corresponds to values $C_{1 / 2}<0.143$, where data falls below the resolution cut-off at $C C^{\star}=0.5$.

results ${ }^{42}$. Preferred crystal orientation limiting data set completeness is often encountered in $\mathrm{ED}^{43}$, even when merging a moderately large number of rotation diffraction data sets ${ }^{29}$. In SerialED, with varying rotation angles as described above, the much higher number of merged crystals, which may occasionally assume unusual orientations, can lead to a mitigation of this issue (see Supplementary Discussion). A further improvement would be achieved using specialized TEM grids ${ }^{44}$, or microfabricated chips ${ }^{45}$. The SerialED approach could also be applied to heterogeneous systems with extended amorphous regions, such as cells containing in vivo grown nanocrystals ${ }^{3}$, or to map and exploit local lattice structures ${ }^{46}$. Similarly, mixtures of crystals within a single grid or contaminated samples can be studied without significant modifications by assigning each found lattice to one of the contained sample classes using multiple indexing runs or direct classification of diffraction patterns ${ }^{36,47}$. It is moreover not only limited to proteins but encompasses all nanocrystalline compounds, such as pharmaceuticals ${ }^{47,48}$ or porous materials $23,49,50$. Augmenting parallel-beam crystallography with coherent scanning diffraction techniques such as convergent-beam diffraction or low-dose ptychography might be a viable way to obtain Bragg reflection phase information ${ }^{51,52}$. Finally, integrating the serial acquisition approach with emerging methods of in situ and time-resolved $\mathrm{EM}^{53-55}$ may open up avenues for roomtemperature structures or structural dynamics studies on beamsensitive systems. All of this makes STEM-based SerialED a versatile, highly efficient and low-cost alternative to canonical structure determination approaches for proteins and beyond.

\section{Methods}

Sample preparation. Commercially available Cydia pomonella granulovirus of formulation Madex Max was obtained from Andermatt Biocontrol. The occlusion bodies were purified from the aqueous suspension by iterative washing and centrifugation cycles. The pellet was then re-suspended in ultra-pure water at $\mathrm{pH} 7$ and subjected to filtration steps through a sequence of nylon mesh filters with decreasing mesh diameter $(100,50,20,10$, and $5 \mu \mathrm{m}$, all Sysmex, Germany) and finally twice through $0.5 \mu \mathrm{m}$ stainless steel filters (IDEX, USA). To increase the concentration of $\mathrm{OBs}$, the suspension was subjected to centrifugation at $21,000 \times g$, and $90 \%$ of the supernatant removed.

HEWL was purchased from Sigma-Aldrich as a lyophilized powder. It was dissolved in $20 \mathrm{mM}$ NaAcetate, $\mathrm{pH} 4.7$, to a concentration of $80 \mathrm{mg} / \mathrm{ml}$. HEWL crystals were grown via batch crystallization, whereby equal volumes of the protein solution and $80 \mathrm{mg} / \mathrm{ml} \mathrm{NaCl}$ were added. Crystals ranging from 5 to $10 \mu \mathrm{m}$ rapidly formed within $2-3 \mathrm{~h}$. The resulting crystal mixture was centrifuged down and $75 \%$ of the supernatant was removed creating a dense crystal slurry. Subsequent vortexing with steel beads in a microfuge tube for $30 \mathrm{~min}$ resulted in a concentrated suspension of crystal fragments in the sub-500 $\mathrm{nm}$ size range.

For each of the above suspensions, $2 \mu \mathrm{l}$ were applied to 400 -mesh carbon grids (type S160-4 purchased from Plano GmbH), whereupon blotting and vitrification using a mixture of liquid ethane/propane was performed in a Vitrobot Mark IV (Thermo Fisher Scientific).

Diffraction data acquisition. All data have been acquired on a Philips Tecnai F20 TWIN S/TEM, equipped with a Gatan 626 cryo-transfer holder, a X-Spectrum Lambda 750k pixel array detector based on a $6 \times 2$ Medipix 3 array $^{25}$, and a custombuilt arbitrary pattern generator addressing the deflector coil drivers, based on National Instruments hardware (see Supplementary Methods for discussion of hardware requirements). Initially, the grids were screened in low-magnification STEM mode for regions exhibiting a high crystal density without excessive overlap and aggregation. While software such as SerialEM ${ }^{56}$ could be used in a straightforward manner to automate this screening step, this was not required for our test samples, as sufficiently homogeneous regions were readily found. 
Table 1 Data collection and refinement statistics.

\begin{tabular}{|c|c|c|}
\hline Parameter & Granulovirus & Lysozyme \\
\hline $\begin{array}{l}\text { Electron energy/ } \\
\text { wavelength }\end{array}$ & $200 \mathrm{kV} / 0.025 \AA$ & $200 \mathrm{kV} / 0.025 \AA$ \\
\hline Dose (fluence) per crystal & $4.7 e^{-/ \AA^{2}}$ & $2.6 \mathrm{e}^{-/ \AA^{2}}$ \\
\hline Space group & 123 & $P 4_{3} 2_{1} 2$ \\
\hline \multicolumn{3}{|l|}{ Unit cell } \\
\hline$a, b, c$ & $103.2,103.2,103.2 \AA$ & 79.1, 79.1, $38 \AA$ \\
\hline$\alpha, \beta, \gamma$ & $90^{\circ}, 90^{\circ}, 90^{\circ}$ & $90^{\circ}, 90^{\circ}, 90^{\circ}$ \\
\hline No. of hits/indexed lattices & $20,608 / 17,974$ & $1,339 / 1,051$ \\
\hline Resolution range & $\begin{array}{l}72.97-1.55 \\
(1.61-1.55) \AA\end{array}$ & $\begin{array}{l}55.93-1.80 \\
(1.86-1.80) \AA\end{array}$ \\
\hline Unique reflections & $25,971(2,577)$ & $15,284(927)$ \\
\hline Multiplicity & $495(70)$ & $26(7)$ \\
\hline Completeness (\%) & $100(100)$ & $77.7(50.4)$ \\
\hline$R_{\text {split }}(\%)$ & $8.7(168.6)$ & $16.7(28.3)$ \\
\hline Mean $I / \sigma(I)$ & $9.22(0.71)$ & $6.72(4.64)$ \\
\hline $\mathrm{CC}_{1 / 2}$ & $1.00(0.19)$ & $0.94(0.22)$ \\
\hline $\mathrm{CC}^{\star}$ & $1.00(0.56)$ & $0.98(0.60)$ \\
\hline Wilson $B$-factor $\left(\AA^{2}\right)$ & 12.3 & 9.59 \\
\hline $\begin{array}{l}\text { Reflections used in } \\
\text { refinement }\end{array}$ & $26,635(2,630)$ & $9,067(569)$ \\
\hline Reflections used for $R$-free & $1297(149)$ & $436(25)$ \\
\hline$R_{\text {work }}(\%)$ & $17.1(32.6)$ & $27.1(37.2)$ \\
\hline$R_{\text {free }}(\%)$ & $19.7(35.5)$ & $31.6(38.6)$ \\
\hline $\begin{array}{l}\text { Number of non- } \\
\text { hydrogen atoms }\end{array}$ & 2,128 & 1,069 \\
\hline Macromolecules & 2,038 & 1,009 \\
\hline Solvent & 90 & 60 \\
\hline RMS bonds/angles & $0.012 \AA / 1.13 \AA$ & $0.003 \AA / 0.47^{\circ}$ \\
\hline $\begin{array}{l}\text { Ramachandran } \\
\text { favored (\%) }\end{array}$ & 97.1 & 96.1 \\
\hline $\begin{array}{l}\text { Ramachandran } \\
\text { allowed (\%) }\end{array}$ & 2.90 & 3.94 \\
\hline $\begin{array}{l}\text { Ramachandran } \\
\text { outliers (\%) }\end{array}$ & 0.41 & 0.00 \\
\hline Rotamer outliers (\%) & 0.45 & 0.94 \\
\hline Clashscore & 1.74 & 7.58 \\
\hline Average $B$-factor & 19.3 & 9.5 \\
\hline Macromolecules & 19.2 & 9.5 \\
\hline Solvent & 20.4 & 9.9 \\
\hline PDB-ID & $6 \mathrm{~S} 2 \mathrm{O}$ & $6 \mathrm{~S} 2 \mathrm{~N}$ \\
\hline
\end{tabular}

Highest-shell values are shown within parentheses.

After screening, the microscope was set to standard STEM mode at the lowest possible magnification, corresponding to a $(18 \mu \mathrm{m})^{2}$ field of view. To achieve a high-current $(\approx 0.1 \mathrm{nA})$, small $(\approx 110 \mathrm{~nm})$, and collimated $(\ll 0.5 \mathrm{mrad})$ nanobeam, the following microscope settings were made: field-emission gun parameters at weakest excitation of both gun lens and $\mathrm{C} 1$ condenser lens (Spot size), disabled mini-condenser (nanoprobe mode), and small $(5 \mu \mathrm{m})$ condenser $(\mathrm{C} 2)$ aperture. The microscope remains in diffraction mode at all times, that is, the back-focal plane of the objective lens is conjugate with the detector.

At each of the identified sample regions, the two-step acquisition sequence as shown in Fig. 1 was performed:

1. The beam was focused on the sample (Fig. 4a), and an overview STEM image of $1024 \times 1024$ pixel resolution was taken across the entire field of view (Fig. 1a) using the high-angle annular dark field detector. The dwell time was set such that the exposure dose remained small, well below $0.1 \mathrm{e}$ $-/ \AA^{2}$. From this image, crystals were automatically identified using standard feature extraction methods, and a list of scan points, corresponding to discrete values of the microscope's scan coil currents, was derived (see Supplementary Methods and Supplementary Fig. 1).

2. The beam was defocused into a collimated nanobeam (Köhler illumination) of $110 \mathrm{~nm}$ diameter ${ }^{40}$, yielding sharp diffraction patterns in the objective back-focal plane and on the detector (Fig. 4b). The actual diffraction data acquisition was then performed by sequentially moving the beam to each of the crystal coordinates using the STEM deflectors and recording a diffraction movie (dose-fractionated data stack) at each position (Figs. 1b and 3).
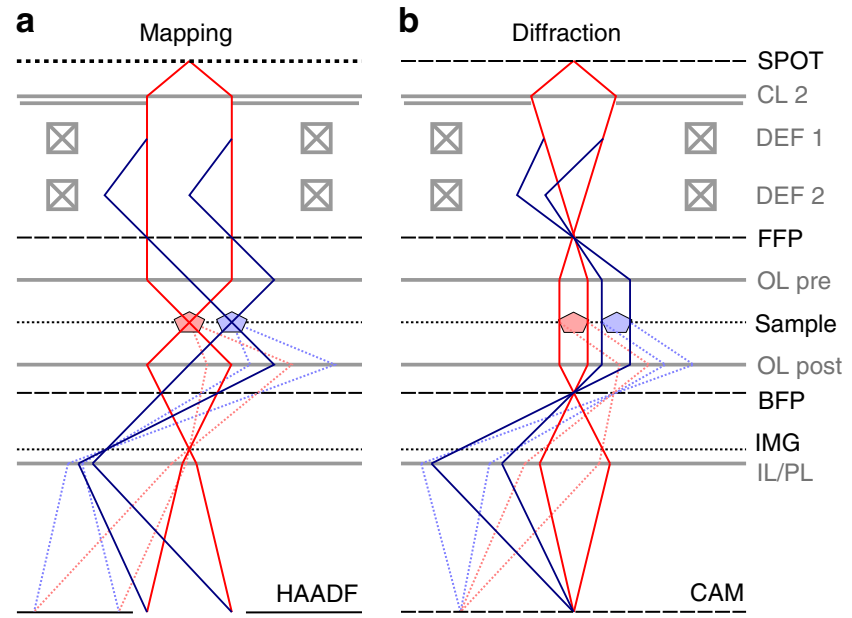

Fig. 4 Ray path diagrams for mapping (focused) and diffraction (collimated) condenser configuration. Red and blue lines correspond to on-axis and one exemplary off-axis positions of the beam. Dotted lines correspond to a Bragg reflection. Optical planes and electron-optical elements are shown in black and gray, respectively. a In the mapping configuration, the beam is collimated by the lower condenser lens (CL 2) and focused on the sample using the objective lens pre-field (OL pre). Scattered beams from the illuminated sample position are imaged on the high-angle annular dark field (HAADF) detector using the objective lens post-field (OL post) and the intermediate and projection lenses (IL/PL). b In the diffraction configuration, on the other hand, the condenser focuses the beam on the front-focal plane (FFP) of the objective. Diffraction orders now appear as discrete spots on the diffraction detector (CAM). Note that switching between these configurations involves changing of the $C L 2$ excitation only, as the detectors always remain in a plane conjugate with the back and front-focal planes of the objective lens (diffraction mode). SPOT-first condenser lens (spot) crossover; DEF1/2-upper and lower beam deflector pair; IMG-intermediate image plane.

Once data acquisition from the mapping region was complete, the beam was blanked, and the sample stage moved to the next previously identified sample region. This sequence was repeated until several thousand diffraction patterns had been recorded. All steps were automated and controlled using Jupyter notebooks based on Python 3.6, and a custom instrument control library written in LabVIEW and Python 3.6, using parts of the Instamatic library ${ }^{38}$.

Data processing. The recorded diffraction patterns were pre-processed using our diffractem package (www.github.com/robertbuecker/diffractem), setting up a pipeline comprising dead-pixel and flat-field detector correction, and partial summing of dose-fractionation stacks, as well as centering of each pattern using the position of the transmitted beam and position matching of simultaneously excited Friedel-mate reflections (Supplementary Fig. 3). Diffraction spots were identified using the peakfinder8 algorithm contained in the CrystFEL suite ${ }^{57,58}$; patterns containing more than 25 spots at resolutions below $\approx 2.5 \AA$ were selected for further analysis. The extracted spot positions were used to determine the orientation of each crystal, and to predict the position of the corresponding Bragg reflections using the indexing and refinement algorithm PinkIndexer ${ }^{39}$. Intensities of the Bragg reflections were extracted using background-corrected pixel summation, and a full reciprocal-space data set was obtained using the partialator program from CrystFEL ${ }^{59}$. Data were truncated after the last resolution shell where $\mathrm{CC}^{*}>0.5^{41,60}$ Refer to Supplementary Methods for additional details on data pre-processing, indexing, and merging. Unmerged and merged reflection intensities in CrystFEL format (stream/hkl) are provided as Supplementary Data 1 (granulin) and Supplementary Data 2 (lysozyme). Phasing of the models was achieved by molecular replacement using Phaser $^{61}$ from the PHENIX software suite ${ }^{62}$ using PDB-ID: 4ET8 and PDB-ID: 5G3X as template models, respectively. Upon obtaining phases, iterative cycles of model building were made using $\operatorname{Coot}^{63}$. For correct refinement of the Coulomb potential maps, subsequent rounds of refinement were performed using phenix.refine, taking electron scattering factors into account ${ }^{64}$. Illustrations of the electron density map and model were generated using PyMOL by Schrödinger. Crystallographic statistics are reported in Table 1. To validate the consistency of our structures with known data, we calculate the root mean square deviations (RMSDs) of atom positions with respect to previously published structures. For Lys with respect to PDB-ID 5K7O (measured by MicroED), we find 
a value of RMSD $=0.487 \AA$; for Lys w.r.t. 5WR9 (measured by XFEL serial crystallography), RMSD $=0.353 \AA$. For GV with respect to PDB-ID 5G3X (synchrotron crystallography), we find $\mathrm{RMSD}=0.206 \AA$; for $\mathrm{GV}$ w.r.t. $5 \mathrm{GOZ}$ (XFEL), $\mathrm{RMSD}=0.353 \AA$.

Reporting summary. Further information on research design is available in the Nature Research Reporting Summary linked to this article.

\section{Data availability}

An example subset of raw experimental data is available from the MPG Open Access Data Repository at https://doi.org/10.17617/3.2j. The full raw data sets are available from R.B. upon request. CrystFEL stream and $h k l$ files containing unmerged and merged reflection intensities, respectively, are available as Supplementary Data 1 (granulin) and Supplementary Data 2 (lysozyme). The protein structures can be accessed from wwPDB using the codes $6 \mathrm{~S} 2 \mathrm{O}$ (granulin) and 6S2N (lysozyme).

\section{Code availability}

PinkIndexer and CrystFEL are available at http://www.desy.de/ twhite/crystfel/ under the terms of the GNU general public license. diffractem is available at www.github.com/ robertbuecker/diffractem under the terms of the MIT license. Python-based Jupyter notebooks executing the data analysis pipeline using diffractem and CrystFEL are available along with the example raw data from the MPG Open Access Data Repository at https://doi.org/10.17617/3.2j. All custom software for data acquisition and hardware control is available from R.B. upon request.

Received: 12 November 2019; Accepted: 27 January 2020;

Published online: 21 February 2020

\section{References}

1. Henderson, R. The potential and limitations of neutrons, electrons and X-rays for atomic resolution microscopy of unstained biological molecules. Q. Rev. Biophys. 28, 171-193 (1995).

2. Glaeser, R. M. How good can single-particle cryo-EM become? What remains before it approaches its physical limits? Annu. Rev. Biophys. 48, 45-61 (2019).

3. Schönherr, R., Rudolph, J. M. \& Redecke, L. Protein crystallization in living cells. Biol. Chem. 399, 751-772 (2018).

4. Owen, R. L., Rudino-Pinera, E. \& Garman, E. F. Experimental determination of the radiation dose limit for cryocooled protein crystals. Proc. Natl Acad. Sci. USA 103, 4912-4917 (2006).

5. Chapman, H. N. et al. Femtosecond X-ray protein nanocrystallography. Nature 470, 73-77 (2011).

6. Tenboer, J. et al. Time-resolved serial crystallography captures high-resolution intermediates of photoactive yellow protein. Science 346, 1242-1246 (2014).

7. Gati, C. et al. Atomic structure of granulin determined from native nanocrystalline granulovirus using an X-ray free-electron laser. Acta Crystallogr. Sect. A 73, a292-a293 (2017).

8. Wiedorn, M. O. et al. Megahertz serial crystallography. Nat. Commun. 9, 4025 (2018).

9. Grünbein, M. L. et al. Megahertz data collection from protein microcrystals at an X-ray free-electron laser. Nat. Commun. 9, 1-9 (2018).

10. Boutet, $\mathrm{S}$. et al. High-resolution protein structure determination by serial femtosecond crystallography. Science 337, 362-364 (2012).

11. Stellato, F. et al. Room-temperature macromolecular serial crystallography using synchrotron radiation. IUCrJ 1, 204-212 (2014).

12. Owen, R. L. et al. Low-dose fixed-target serial synchrotron crystallography. Acta Crystallogr. Sect. D 73, 373-378 (2017).

13. Gati, C. et al. Serial crystallography on in vivo grown microcrystals using synchrotron radiation. IUCrJ 1, 87-94 (2014).

14. Schulz, E. C. et al. The hit-and-return system enables efficient time-resolved serial synchrotron crystallography. Nat. Methods 15, 901-904 (2018).

15. Mehrabi, P. et al. Time-resolved crystallography reveals allosteric communication aligned with molecular breathing. Science 365, 1167-1170 (2019).

16. Mehrabi, P. et al. Liquid application method for time-resolved analyses by serial synchrotron crystallography. Nat. Methods 16, 979-982 (2019).

17. Beyerlein, K. R. et al. Mix-and-diffuse serial synchrotron crystallography. IUCrJ 4, 769-777 (2017).

18. Ebrahim, A. et al. Dose-resolved serial synchrotron and XFEL structures of radiation-sensitive metalloproteins. IUCrJ 6, 543-551 (2019).

19. Clabbers, M. T. B. \& Abrahams, J. P. Electron diffraction and threedimensional crystallography for structural biology. Crystallogr. Rev. 24, 176-204 (2018).
20. Henderson, R. \& Unwin, P. N. T. Three-dimensional model of purple membrane obtained by electron microscopy. Nature 257, 28-32 (1975).

21. Gemmi, M. et al. 3D electron diffraction: the nanocrystallography revolution. ACS Cent. Sci. 5, 1315-1329 (2019).

22. Mugnaioli, E., Gorelik, T. \& Kolb, U. "Ab initio" structure solution from electron diffraction data obtained by a combination of automated diffraction tomography and precession technique. Ultramicroscopy 109, 758-765 (2009).

23. Zhang, Y. et al. Single-crystal structure of a covalent organic framework. J. Am. Chem. Soc. 135, 16336-16339 (2013).

24. Shi, D., Nannenga, B. L., Iadanza, M. G. \& Gonen, T. Three-dimensional electron crystallography of protein microcrystals. Elife 2, e01345 (2013).

25. Nederlof, I., van Genderen, E., Li, Y.-W. \& Abrahams, J. P. A Medipix quantum area detector allows rotation electron diffraction data collection from submicrometre three-dimensional protein crystals. Acta Crystallogr. Sect. D 69, 1223-1230 (2013)

26. Nannenga, B. L., Shi, D., Leslie, A. G. W. \& Gonen, T. High-resolution structure determination by continuous-rotation data collection in microEDED. Nat. Methods 11, 927-930 (2014).

27. Yonekura, K., Kato, K., Ogasawara, M., Tomita, M. \& Toyoshima, C. Electron crystallography of ultrathin 3D protein crystals: atomic model with charges. Proc. Natl Acad. Sci. USA 112, 3368-3373 (2015).

28. Clabbers, M. T. B. et al. Protein structure determination by electron diffraction using a single three-dimensional nanocrystal. Acta Crystallogr. Sect. D 73, 738-748 (2017).

29. $\mathrm{Xu}, \mathrm{H}$. et al. A rare lysozyme crystal form solved using highly redundant multiple electron diffraction datasets from micron-sized crystals. Structure $\mathbf{2 6}$, 667-675.e3 (2018).

30. Lanza, A. et al. Nanobeam precession-assisted 3D electron diffraction reveals a new polymorph of hen egg-white lysozyme. IUCrJ 6, 178-188 (2019).

31. $\mathrm{Xu}, \mathrm{H}$. et al. Solving a new R2lox protein structure by microcrystal electron diffraction. Sci. Adv. 5, 1-7 (2019).

32. Kolb, U., Gorelik, T., Kübel, C., Otten, M. T. \& Hubert, D. Towards automated diffraction tomography: part I-data acquisition. Ultramicroscopy 107, 507-513 (2007).

33. Cichocka, M. O., Ångström, J., Wang, B., Zou, X. \& Smeets, S. Highthroughput continuous rotation electron diffraction data acquisition via software automation. J. Appl. Crystallogr 51, 1652-1661 (2018).

34. de la Cruz, M. J., Martynowycz, M. W., Hattne, J. \& Gonen, T. MicroED data collection with SerialEM. Ultramicroscopy 201, 77-80 (2019).

35. Yonekura, K., Ishikawa, T. \& Maki-Yonekura, S. A new cryo-EM system for electron 3D crystallography by eEFD. J. Struct. Biol. 206, 243-253 (2019).

36. Wang, B., Zou, X. \& Smeets, S. Automated serial rotation electron diffraction combined with cluster analysis: an efficient multi-crystal workflow for structure determination. IUCrJ 6, 854-867 (2019).

37. Hattne, J. et al. Analysis of global and site-specific radiation damage in cryoEM. Structure 26, 759-766.e4 (2018).

38. Smeets, S., Zou, X. \& Wan, W. Serial electron crystallography for structure determination and phase analysis of nanocrystalline materials. J. Appl. Crystallogr 51, 1-12 (2018).

39. Gevorkov, Y. et al. pinkIndexer-a universal indexer for pink-beam X-ray and electron diffraction snapshots. Acta Crystallogr. Sect. A $\mathbf{7 6}$ https://doi.org/ $10.1107 /$ S2053273319015559 (2020).

40. He, H. \& Nelson, C. A method of combining STEM image with parallel beam diffraction and electron-optical conditions for diffractive imaging. Ultramicroscopy 107, 340-344 (2007).

41. Karplus, P. A. \& Diederichs, K. Linking crystallographic model and data quality. Science 336, 1030-1033 (2012).

42. Jiang, L., Georgieva, D., Zandbergen, H. W. \& Abrahams, J. P. Unit-cell determination from randomly oriented electron-diffraction patterns. Acta Crystallogr. Sect. D 65, 625-632 (2009).

43. Nannenga, B. L., Shi, D., Hattne, J., Reyes, F. E. \& Gonen, T. Structure of catalase determined by microED. Elife 3, e03600 (2014).

44. Wennmacher, J. T. C. et al. 3D-structured supports create complete data sets for electron crystallography. Nat. Commun. 10, 1-6 (2019).

45. Mueller, C. et al. Fixed target matrix for femtosecond time-resolved and in situ serial micro-crystallography. Struct. Dyn. 2, 054302 (2015).

46. Gallagher-Jones, M. et al. Nanoscale mosaicity revealed in peptide microcrystals by scanning electron nanodiffraction. Commun. Biol. 2, 26 (2019).

47. Jones, C. G. et al. The CryoEM method MicroED as a powerful tool for small molecule structure determination. ACS Cent. Sci. 4, 1587-1592 (2018).

48. Gruene, T. et al. Rapid structure determination of microcrystalline molecular compounds using electron diffraction. Angew. Chem. Int. Ed. 57, 16313-16317 (2018).

49. Jiang, J. et al. Synthesis and structure determination of the hierarchical mesomicroporous zeolite ITQ-43. Science 333, 1131-1134 (2011). 
50. Denysenko, D. et al. Elucidating gating effects for hydrogen sorption in MFU4-type triazolate-based metal-organic frameworks featuring different pore sizes. Chem. A Eur. J. 17, 1837-1848 (2011).

51. Pelz, P. M., Qiu, W. X., Bücker, R., Kassier, G. \& Miller, R. J. D. Low-dose cryo electron ptychography via non-convex Bayesian optimization. Sci. Rep. 7, 9883 (2017).

52. Ophus, C. Four-dimensional scanning transmission electron microscopy (4DSTEM): from scanning nanodiffraction to ptychography and beyond. Microsc. Microanal. 25, 563-582 (2019).

53. Karakulina, O. M., Demortière, A., Dachraoui, W., Abakumov, A. M. \& Hadermann, J. In situ electron diffraction tomography using a liquidelectrochemical transmission electron microscopy cell for crystal structure determination of cathode materials for Li-ion batteries. Nano Lett. 18, 6286-6291 (2018).

54. Kaledhonkar, S. et al. Late steps in bacterial translation initiation visualized using time-resolved cryo-EM. Nature 570, 400-404 (2019).

55. Ross, F. M. Opportunities and challenges in liquid cell electron microscopy. Science 350, aaa9886-aaa9886 (2015).

56. Mastronarde, D. N. Automated electron microscope tomography using robust prediction of specimen movements. J. Struct. Biol. 152, 36-51 (2005).

57. White, T. A. et al. CrystFEL: a software suite for snapshot serial crystallography. J. Appl. Crystallogr 45, 335-341 (2012).

58. Barty, A. et al. Cheetah: software for high-throughput reduction and analysis of serial femtosecond X-ray diffraction data. J. Appl. Crystallogr. 47, 1118-1131 (2014).

59. White, T. A. et al. Recent developments in CrystFEL. J. Appl. Crystallogr. 49, 680-689 (2016).

60. Diederichs, K. Dissecting random and systematic differences between noisy composite data sets. Acta Crystallogr. Sect. D 73, 286-293 (2017).

61. McCoy, A. J. et al. Phaser crystallographic software. J. Appl. Crystallogr. 40, 658-674 (2007).

62. Adams, P. D. et al. PHENIX: a comprehensive Python-based system for macromolecular structure solution. Acta Crystallogr. Sect. D 66, 213-221 (2010).

63. Emsley, P., Lohkamp, B., Scott, W. G. \& Cowtan, K. Features and development of Coot. Acta Crystallogr. Sect. D 66, 486-501 (2010).

64. Afonine, P. V. et al. Towards automated crystallographic structure refinement with phenix.refine. Acta Crystallogr. Sect. D 68, 352-367 (2012).

\section{Acknowledgements}

We thank Djordje Gitaric for mechanical design work, Fabian Westermeier, David Pennicard, and Heinz Graafsma for adapting the Lambda detector for electron imaging, Anton Barty and Henry Chapman for many helpful discussions and critical reading of the manuscript, Thomas A. White for help with modifying CrystFEL for electron diffraction, and Michiel de Kock for help with image processing. We are indebted to Kay Grünewald and his research group for lending to us their cryo-transfer holder. We gratefully acknowledge the support provided by the Max Planck Society, Deutsches Elektronen-Synchrotron (DESY), the excellence cluster "The Hamburg Center for Ultrafast Imaging" of the Deutsche Forschungsgemeinschaft (EXC 1074 project ID
194651731), the European Research Council project “Attosecond X-ray Science: Imaging and Spectroscopy" (Award/Contract Number ERC-2013-SyG 609920), and the Joachim Herz Foundation (Biomedical Physics of Infection). P.H. acknowledges support by the Natural Sciences and Engineering Research Council of Canada. P.M. was supported by the Alexander von Humboldt-Stiftung for postdoctoral researchers.

\section{Author contributions}

R.B., G.H.K., and R.J.D.M. conceived the SerialED scheme. R.B. implemented the experimental setup and conducted the measurements. P.M. and G.H.K. prepared the lysozyme nanocrystals, D.O. prepared the granulovirus sample, and G.H.K. and L.A.B. prepared vitrified cryo-EM grids from each sample. R.B., P.H.-L., and P.M. performed the data processing. Y.G., O.Y., and W.B. adapted the PinkIndexer code to electron diffraction. R.B., E.C.S., and G.H.K. wrote the paper, with contributions from all authors.

\section{Competing interests}

The authors declare no competing interests.

\section{Additional information}

Supplementary information is available for this paper at https://doi.org/10.1038/s41467020-14793-0.

Correspondence and requests for materials should be addressed to R.J.D.M.

Peer review information Nature Communications thanks the anonymous reviewer(s) for their contribution to the peer review of this work.

Reprints and permission information is available at http://www.nature.com/reprints

Publisher's note Springer Nature remains neutral with regard to jurisdictional claims in published maps and institutional affiliations.

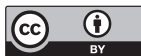

Open Access This article is licensed under a Creative Commons Attribution 4.0 International License, which permits use, sharing, adaptation, distribution and reproduction in any medium or format, as long as you give appropriate credit to the original author(s) and the source, provide a link to the Creative Commons license, and indicate if changes were made. The images or other third party material in this article are included in the article's Creative Commons license, unless indicated otherwise in a credit line to the material. If material is not included in the article's Creative Commons license and your intended use is not permitted by statutory regulation or exceeds the permitted use, you will need to obtain permission directly from the copyright holder. To view a copy of this license, visit http://creativecommons.org/ licenses/by/4.0/.

(C) The Author(s) 2020 